José Antonio Menezes Filho' Wilson Andrade de Carvalho Ademário Galvão Spínola ${ }^{3}$

\title{
Avaliação da Exposição Ocupacional ao Chumbo em uma Metalúrgica Um Estudo Transversal
}

\author{
Assessment of Occupational Expo- \\ sure to Lead in a Metallurgy Plant \\ A Cross-Sectional Study
}

'Farmacêutico-Bioquímico, M.Sc. Professor de Toxicologia da Universidade Federal da Bahia e Coordenador do Laboratório de Patologia Clínica e Toxicologia do Hospital São Rafael

${ }^{2}$ Médico da Universidade Federal da Bahia, Faculdade de Farmácia e Chefe do Laboratório de Patologia Clínica e Toxicologia do Hospital São Rafael.

${ }^{3}$ Médico da Universidade Federal da Bahia, Faculdade de Medicina, Departamento de Medicina Preventiva e Chefe do Serviço de Medicina do Trabalho do Hospital São Rafael.
OBJETIVO: Avaliar o nível de exposição ocupacional em uma metalúrgica de chumbo, através da determinação dos indicadores biológicos de exposição e efeito, com o objetivo de estabelecer um mapeamento das áreas de risco dentro da planta industrial. METODOLOGIA: Estudo transversal incluindo 195 trabalhadores de uma metalúrgica primária de chumbo, localizada no Estado da Bahia-Brasil, e um grupo de referência de 65 indivíduos. Nos grupos de expostos e controle foram realizadas as dosagens dos indicadores biológicos de exposição e efeito (IBE's) ao chumbo inorgânico, incluindo Pb-S, ALA-U, ZnPP e Hb.

Palavras-chaves Chumbo Sangüíneo, Exposição Ocupacional, ALA, PB-S.

OBJECTIVE: The aim of this study was the assessment of occupational exposure to lead in a metallurgy plant, by measuring biological exposure and effects indicators; and the mapping of risk areas throughout the industrial plant. METHODOLOGY: A cross-sectional study was performed involving 195 workers at a lead metallurgy plant and a reference group of 65 individuals, in the State of Bahia, Brazil. Both the exposed and control groups were tested for dosage levels for biological exposure and effects indicators for inorganic lead, including $\mathrm{Pb}-\mathrm{S}, \mathrm{ALA}-\mathrm{U}, \mathrm{ZnPP}$ and $\mathrm{Hb}$ - (blood lead level (BLL); $\mathrm{d}$-aminolevulinic acid in urine (ALA-U); zinc-protoporphyrin (Zn-PP); and hemoglobin (Hb).

Keywords Lead in Blood, Occupational Exposure, BLL, ALA. 


\section{Introdução}

O chumbo é um metal com uma longa história de uso industrial e com importantes efeitos tóxicos sobre a saúde humana. Estudos epidemiológicos realizados em diversos países do mundo revelam inúmeros casos de intoxicação, notadamente em exposições ocupacionais e ambientais. Apesar do extraordinário conhecimento adquirido nas últimas décadas sobre o mecanismo de toxicidade do metal, das recentes medidas de prevenção e de supervisão médica e principalmente da aplicação de eficientes medidas de higiene industrial, concorrendo para uma considerável redução nos níveis de exposição ocupacional, lamentavelmente ainda continua ocorrendo casos de intoxicação ${ }^{1,2,3,4}$. Elevada exposição ao chumbo e casos de intoxicação têm sido encontrados, notadamente em crianças fiIhas de trabalhadores de fundição de chumbo e em crianças residentes próximo às fundições primárias de chumbo 5,6. Mesmo em áreas sem fonte pontual de emissão de chumbo no meio ambiente, pode haver a contaminação dos seres vivos. Alguns estudos demonstraram a contaminação, principalmente de crianças, através do material particulado em casario antigo pintado com tinta a base de chumbo, proximidade de autoestrada e do contato direto com o solo $0^{7-9}$.

As principais fontes de exposição ocupacional ao chumbo são representadas principalmente pelas minerações, fundições e refinarias de chumbo, fábricas de baterias (acumuladores), indústria cerâmica e fundições de metais não ferrosos ${ }^{10-13}$, encontradas com grande freqüência em diversos estados brasileiros, inclusive na Bahia.

Os processos de fundição e refinaria, são provavelmente, entre todas as modalidades de exposição ocupacional, as que apresentam maior risco de exposição ao metal, provavelmente porque envolvem operações nas quais o chumbo fundido e as ligas são aquecidas a temperaturas elevadas com desprendimento de vapores de chumbo ${ }^{10}$, 12,14 .

No ambiente laboral a absorção do chumbo resulta principalmente da inalação de pequenas partículas de tamanho respirável ou dos fumos do metal. Secundariamente, pode ocorrer absorção através da ingestão de alimentos contaminados, água e por hábitos do trabalho, como o de fumar ${ }^{10,12}$ e por contaminação dérmica e do vestuário.

Diversos estudos epidemiológicos e toxicológicos têm sido realizados recentemente em populações com exposição ocupacional ao chumbo com o objetivo de desenvolver marcadores biológicos de toxicidade subclínica adequados para a aplicação em programas de saúde ocupacional, particularmente para baixos níveis de exposição ${ }^{3,12,15}$. Os Indicadores Biológicos de Exposição (IBE) mais comumente empregados e recomendados para a monitorização biológica da exposição ocupacional ao chumbo são representados principalmente pelo chumbo no sangue $(\mathrm{Pb}-\mathrm{S})$, zinco-protoporfirina ( $\mathrm{ZnPP})$ e ácido d-aminolevulínico na urina (ALA-U). Outros indicadores como ácido $d$ aminolevulínico desidratase (ALA-D) e a coproporfirina urinária (CP-U), não são comumente empregados em saúde ocupacional, principalmente por apresentarem pouca especificidade 11, 14, 16-22.

O objetivo deste estudo foi avaliar o nível de exposição em uma metalúrgica de chumbo, através da determinação dos indicadores biológicos de exposição e efeito do chumbo, a fim de estabelecer um mapeamento das áreas de risco na planta industrial.

\section{Material e métodos Caracterização dos Grupos}

Foram investigados cento e noventa e cinco trabalhadores adultos do sexo masculino de uma refinaria primária de chumbo localizada no Estado da Bahia, Brasil no ano de 1993. De acordo com o fluxograma do processo de produção da planta industrial e de organização e distribuição de cargos e funções da empresa, os trabalhadores foram distribuídos nos seguintes grupos de atividade laboral: sinterização $(n=23)$, fusão $(n=41)$, refinaria $(n=49)$, laboratório $(n=8)$, serviços gerais $(n=17)$, manutenção $(n=26)$ e administração $(n=$ 31). Os grupos de sinterização, fusão e refinaria exerciam suas atividades laborais nos respectivos setores industriais, os indivíduos do grupo de serviços gerais, com atividades de limpeza; e os da manutenção, 
com atividades de manutenção dentro da planta. O pessoal de laboratório participava da execução, coleta e determinação analítica das amostras destinadas ao controle de qualidade de produção e da monitorização ambiental nos programas de higiene industrial da empresa. $\mathrm{O}$ grupo controle foi constituído por 65 indivíduos pareados por sexo, idade, raça, hábitos alimentares, tabagismo e alcoolismo com o grupo de trabalhadores expostos na planta industrial, diferindo apenas quanto ao aspecto de não se referirem a exposição atual ou no passado aos compostos de chumbo.

\section{Metodologia}

Foi realizado um estudo de corte transversal incluindo os grupos de expostos e controle nos quais foram realizadas as determinações de chumbo no sangue (Pb-S), ácido daminolevulínico na urina (ALA-U), zincoprotoporfirina (ZnPP) e hemoglobina $(\mathrm{Hb})$ no sangue total.

O sangue foi coletado por punção venosa em veia cubital, através de sistema a vácuo, em dois tubos de vidro com EDTA livre de resíduo de chumbo. O tubo de sangue destinado à determinação de chumbo foi armazenado em geladeira a $2-8^{\circ} \mathrm{C}$ até o momento da análise e o tubo coletado para a determinação de $\mathrm{Hb}$ foi imediatamente processado. A urina foi coletada em coletores de polietileno descontaminado com ácido nítrico a $2 \%$ em água pura Tipo I (Sistema Milli-RX45 acoplado a um Milli-Q, Millipore). A concentração de chumbo no sangue foi determinada em duplicata por espectroscopia de absorção atômica, usando o método eletrotémico de digestão da amostra em forno de grafite, segundo o método de Fernadez modificado ${ }^{23}$, utilizando o equipamento Perkin-Elmer 5000 equipado com forno de grafite P.E. HGA-500. A dosagem do ALA$U$ foi realizada pelo método espectrofotométrico ${ }^{24}$, usando 0 espectrofotômetro Perkin-Elmer Lambda I. O resultado foi corrigido pelo valor da creatinina urinária, que foi dosada pelo método cinético de Jaffé no analisador bioquímico MEGA da Merck Diagnósticos. A determinação da ZnPP foi executada por fluorimetria direta em sangue total, utilizando-se o hematofluorômetro
Protofluor-Z da Helena Laboratories, seguindo o protocolo do fabricante. A hemoglobina foi determinada imediatamente após a coleta do sangue, usando-se o sistema automatizado STKS da Coulter.

O controle de qualidade analítica para a determinação do $\mathrm{Pb}$-S foi realizado usando o sangue controle fornecido pela Bio-Rad ${ }^{\circledR}$, Liphocheck Nivel I e II, além do controle externo de qualidade do Programa de Intercalibração de Controle de Qualidade do Instituto Nacional de Seguridad y Higiene del Trabajo (Espanha). O controle de qualidade das determinações bioquímicas foi realizado através do programa de Controle Externo de Qualidade da Sociedade Brasileira de Patologia Clínica e SEGULAB (Itália).

Após extratificação por grupos de atividade, as médias de cada foram comparadas através do teste não paramétrico de Wilcoxon para médias não-pareadas, análise de variância one-way (ANOVA) e estudo de correlação, utilizando-se o programa estatístico Epinfo 6.0.

\section{Resultados}

Os resultados das determinações de $\mathrm{Pb}$ $\mathrm{S}, \mathrm{ALA}-\mathrm{U}, \mathrm{ZnPP}$ e Hb , do grupo controle e dos trabalhadores da metalúrgica, estão resumidos na Tabela 1.

Comparando-se os valores de $\mathrm{Pb}-\mathrm{S}$ do grupo controle $(5,4 \pm 3,9 \mu \mathrm{g} / \mathrm{dL})$ com o grupo de indivíduos da área administrativa $(13,1 \pm 8,4 \mu \mathrm{g} / \mathrm{dL})$, constatou-se uma diferença estatisticamente significativa $(p<0,001)$ entre eles, demonstrando que embora os trabalhadores administrativos não exerçam atividade na área de produção da planta, possuíam uma fonte adicional de exposição, oriunda provavelmente, da contaminação ambiental da metalúrgica. Não foi observada diferença significativa $(p>0,05)$ entre os indicadores de efeito (ALA, ZnPP e HB) deste grupo quando comparado com o grupo controle. Diferença ainda mais significativa ( $p<0,0001$ ) foi constatada comparando-se - $\mathrm{Pb}$-S do grupo controle com o $\mathrm{Pb}-\mathrm{S}$ do grupo dos trabalhadores de Serviços Gerais $(25,1 \pm 9,1 \mu \mathrm{g} / \mathrm{dL})$, da Manutenção $(28,5 \pm 9,8 \mathrm{\mu g} / \mathrm{dL})$, do Laboratório $(23,1 \pm 7,6 \mu \mathrm{g} / \mathrm{dL})$ e da Sinterização 
$(36,7 \pm 9,8 \mu \mathrm{g} / \mathrm{dL})$. Entretanto, não foi observada diferença significativa $(p>0,05)$ entre $\circ \mathrm{Pb}$-S dos grupos de Refinaria, Fusão e Sinterização, sugerindo níveis semelhantes de exposição, bem como entre os grupos de Laboratório, Manutenção e Serviços Gerais.Em relação ao ALA-U foi constatada uma diferença altamente significativa $(p<0,0001)$ quando comparado o controle $(1,9 \pm 0,9 \mathrm{mg} / \mathrm{g}$ creat.) com os grupos da Manutenção $(6,9 \pm 3,8 \mathrm{mg} / \mathrm{g}$ creat.),

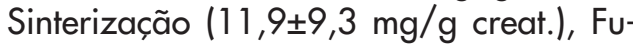
são $(11,1 \pm 6,9 \mathrm{mg} / \mathrm{g}$ creat.) e Refinaria $(10,3 \pm 9,5 \mathrm{mg} / \mathrm{g}$ creat.), não sendo, porém, observada diferença estatística entre as médias destes grupos de trabalhadores. Considerando a ZnPP, constatou-se uma diferença altamente significativa $(p<0,0001)$ entre o grupo controle $(13,5 \pm 0,9 \mu \mathrm{g} / \mathrm{dL})$ e os grupos de Fusão $(65,5 \pm 45,5 \mathrm{\mu g} / \mathrm{dL}), \quad$ Sinterização $(87,5 \pm 107,0 \mathrm{\mu g} / \mathrm{dL})$ e Refinaria $(65,5 \pm 61,5 \mu \mathrm{g} / \mathrm{dL})$. Esta diferença foi menos significativa $(p<0,001)$ para o grupo da Manutenção $(33,0 \pm 38,0 \mu \mathrm{g} / \mathrm{dL})$ e para o grupo de Serviços Gerais $(25,5 \pm 20,0 \mu \mathrm{g} / \mathrm{dL})$. Não sendo observada, porém, diferença significativa $(p>0,05)$ entre os grupos controle $(25,5 \pm 20,0 \mu \mathrm{g} /$ $\mathrm{dL})$ e o da Administração $(21,0 \pm 28,0 \mathrm{\mu g} /$ $d L)$.

Tabela 1 Valores dos Indicadores Biológicos de Efeito e Exposição nos Grupos Expostos e Controle.

\begin{tabular}{|c|c|c|c|c|}
\hline Grupos Avaliados & Pb-S & $\begin{array}{c}\text { ALA-U } \\
\mathrm{mg} / \mathrm{g} \text { creat. }\end{array}$ & $\begin{array}{l}\text { ZnPP } \\
\mathrm{fg} / \mathrm{dL}\end{array}$ & $\begin{array}{l}\mathbf{H b} \\
\mathrm{g} / \mathrm{dL}\end{array}$ \\
\hline $\begin{array}{l}\text { Grupo Controle } \\
n=65\end{array}$ & $\begin{array}{c}5,4 \pm 3,1 \\
(1,4-16,6)\end{array}$ & $\begin{array}{l}1,9 \pm 0,9 \\
(0,5-6,3)\end{array}$ & $\begin{array}{l}16,5 \pm 7,0 \\
(8,5-59)\end{array}$ & $\begin{array}{l}14,0 \pm 1,3 \\
(0,7-17,4)\end{array}$ \\
\hline $\begin{array}{l}\text { Administração } \\
n=31\end{array}$ & $\begin{array}{l}13.1 \pm 8,4^{*} \\
(1,4-34,8)\end{array}$ & $\begin{array}{c}4,6 \pm 3,0 \\
(1,2 \cdot 17,4)\end{array}$ & $\begin{array}{c}21 \pm 28 \\
(5,5-125)\end{array}$ & $\begin{array}{r}14,4 \pm 1,5 \\
(11,6-16,9)\end{array}$ \\
\hline $\begin{array}{l}\text { Ser. Gerais } \\
n=17\end{array}$ & $\begin{array}{l}25,1 \pm 9,1 * \\
(17,7-46,3)\end{array}$ & $\begin{array}{c}6,0 \pm 3,8 \\
(2,5-18,3)\end{array}$ & $\begin{array}{c}25,5 \pm 20 \\
(9-80)\end{array}$ & $\begin{array}{c}15,0 \pm 0,8 \\
(13,5-16,1)\end{array}$ \\
\hline $\begin{array}{l}\text { Manutenção } \\
n=26\end{array}$ & $\begin{array}{l}28,5 \pm 9,8^{*} \\
(10,7-42,6)\end{array}$ & $\begin{array}{l}6,9 \pm 8,4^{*} \\
(1,4-42,1)\end{array}$ & $\begin{array}{c}33 \pm 38 \\
(10,5-150)\end{array}$ & $\begin{array}{c}14,8 \pm 0,9 \\
(13,2-16,1)\end{array}$ \\
\hline $\begin{array}{l}\text { Laboratório } \\
n=8\end{array}$ & $\begin{array}{l}23,1 \pm 7,6^{*} \\
(11,0 \cdot 56,5)\end{array}$ & $\begin{array}{l}4,3 \pm 1,0 \\
(3,3-6,6)\end{array}$ & $\begin{array}{l}25,5 \pm 17 \\
(10 \cdot 63,5)\end{array}$ & $\begin{array}{c}15,6 \pm 0,4 \\
(14,8-16,1)\end{array}$ \\
\hline $\begin{array}{l}\text { Sinterização } \\
n=23\end{array}$ & $\begin{array}{l}36,7 \pm 9,8^{*} \\
(5,6-55,4)\end{array}$ & $\begin{array}{c}11,9 \pm 9,3^{*} \\
(2,54-32,55)\end{array}$ & $\begin{array}{l}87,5 \pm 107^{*} \\
(14,5-458)\end{array}$ & $\begin{array}{c}15,0 \pm 0,9 \\
(13,9-17,0)\end{array}$ \\
\hline $\begin{array}{l}\text { Fusão } \\
n=41\end{array}$ & $\begin{array}{l}37,9 \pm 8,5^{*} \\
(24,8 \cdot 55,0)\end{array}$ & $\begin{array}{l}11,1 \pm 6,9^{*} \\
(2,4-30,9)\end{array}$ & $\begin{array}{c}65,5 \pm 45,5^{*} \\
(11,5 \cdot 150)\end{array}$ & $\begin{array}{c}14,4 \pm 1,2 \\
(11,9-16,9)\end{array}$ \\
\hline $\begin{array}{l}\text { Refinaria } \\
n=49\end{array}$ & $\begin{array}{l}36,0 \pm 9,7^{*} \\
(11,0-56,5)\end{array}$ & $\begin{array}{c}10,3 \pm 9,5^{*} \\
1,2 \cdot 47,0\end{array}$ & $\begin{array}{c}65,5 \pm 61,5^{*} \\
(17-307)\end{array}$ & $\begin{array}{c}15,1 \pm 0,9 \\
(12,9-16,8)\end{array}$ \\
\hline
\end{tabular}

Nota: Os valores são média \pm desvio padrão e amplitude entre parênteses. Os grupos com diferença estatística significativa $(p<0,05)$ do grupo controle estão com asterísco. 


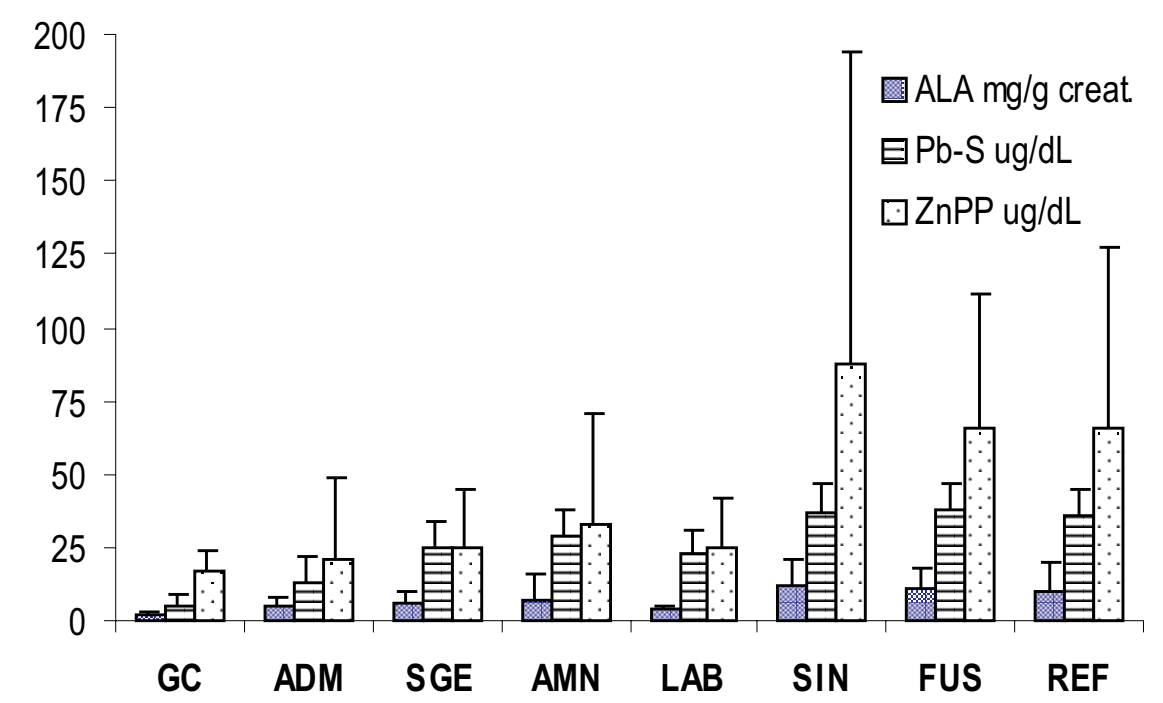

Legenda: Barra representa a média aritmética e travessão representa o desvio padrão.

Figura 1 Distribuição dos bioindicadores PB-S, ALA e ZnPP nos grupos controle e nos grupos de trabalhadores expostos.

A Figura 1 apresenta uma distribuição gráfica dos valores médios de Pb-S, ZnPP e ALAU dos grupos controle e dos grupos de trabalhadores expostos da metalúrgica.

\section{Discussão}

Os níveis de chumbo no sangue na população geral têm diminuído ao longo do tempo em diversos países do mundo, provavelmente resultante de ações de controle de determinadas fontes de emissão de chumbo, como as de origem industrial e de derivados do petróleo. O uso do álcool adicionado à gasolina reduziu a utilização de antidetonante a base de chumbo, contribuindo substancialmente para a redução da emissão atmosférica de chumbo pelos automóveis, como acontece no Brasil|25-27. $\mathrm{Na}$ maioria dos países a concentração média de chumbo no sangue em pessoas não expostas ocupacionalmente está situada entre 10 e $15 \mathrm{ig} / \mathrm{dL}$ e em algumas situações é inferior a $10 \mathrm{ig} / \mathrm{dL}$. Na Suécia a média de $\mathrm{Pb}-\mathrm{S}$ é de $8 \mathrm{ig} / \mathrm{dL}$ no sexo masculino e de 6 ìg/dL para o sexo feminino ${ }^{25}$. Na ltália o nível de $\mathrm{Pb}-\mathrm{S}$ encontrado na população geral é de 12-15 ig/dL para o sexo masculino e de 8-10 ig/dL para o sexo feminino ${ }^{4}$, na Espanha de 7,8 ìg/dL e nos Estados Unidos da América do Norte de 16,1 ig/dL para o sexo masculino e de 11,0 ìg/dL para o sexo feminino ${ }^{28}$. Níveis mais elevados em indivíduos da população geral têm sido observados em outros países, como na Cidade do México onde foram encontrados $25,5 \mathrm{ig} / \mathrm{dL}$ $\mathrm{de} \mathrm{Pb}-\mathrm{S}$ no sexo masculino e 19,3 ig/ $\mathrm{dL}$ no sexo feminino 29 .

Os resultados obtidos do grupo controle, formado por indivíduos adultos do sexo masculino, revelam que a média do $\mathrm{Pb}-\mathrm{S}$ foi de 5,4 ig/dL (Tabela 1), inferior ao valor de 12,4 ig/dL encontrado para a Cidade de São Paulo - Brasil ${ }^{30}$ e dos reportados para a maioria dos países ${ }^{25}$. Os valores de ALA-U, ZNPP e Hb encontrados no grupo controle são semelhantes aos valores de referência destes indicadores observados em populações de indivíduos não ocupacionalmente expostos ${ }^{31,32}$.

O grupo da Administração apresentou $\mathrm{Pb}-\mathrm{S}$ de 13,1 ìg/dL, significativamente mais elevado que o grupo controle $(p<0,001)$, demonstrando que a proximidade do escritório administrativo da área de produção da planta industrial e o uso do mesmo refeitório e de outras instalações da empresa, podem ter contribuído para uma maior 
exposição ambiental deste grupo, passando a apresentar níveis sangüíneos de chumbo semelhantes aos de indivíduos que convivem próximo a áreas industriais de produção de chumbo reportados por outros pesquisadores $^{6,10,25,30}$

Embora tenha ocorrido considerável aumento na produção e consumo dos compostos de chumbo na década de 1970, observou-se paralelamente uma redução substancial nos níveis de $\mathrm{Pb}-\mathrm{S}$ de indivíduos ocupacionalmente expostos, refletindo uma melhoria e maior atenção aos programas de vigilância epidemiológica, de medidas mais eficazes de higiene industrial e de monitorização biológica dos trabalhadores expostos 3, 25,29. Apesar disso, ainda continua ocorrendo em alguns países situações consideradas de elevado risco, como a constatada em 1987 nos Estados Unidos da América do Norte, em que mais de 1.000 trabalhadores tinham $\mathrm{Pb}-\mathrm{S}$ acima de $40 \mathrm{ig} / \mathrm{dL}$ e aproximadamente 200 deles possuíam níveis de chumbo no sangue aci$\mathrm{ma}$ de $50 \mathrm{ig} / \mathrm{dL}$. Verificou-se neste e em outros estudos que a maioria dos trabalhadores com concentração mais elevada exerciam atividade em mineração, refinaria, fundições e em indústrias de acumuladores. Os processos de fundição e refinaria do chumbo são provavelmente os que representam maior risco de exposição, confirmando os resultados obtidos neste estudo no qual os trabalhadores das áreas de refinaria, fusão e sinterização apresentaram as concentrações mais elevadas de chumbo no sangue ${ }^{1,3}, 10,25,33,34$.

Em 1980 a Organização Mundial de Saúde ${ }^{25}$ recomendou como limite de tolerância biológico (LTB) para exposição ocupacional ao chumbo o $\mathrm{Pb}-\mathrm{S}$ de $40 \mathrm{ig} /$ $\mathrm{dL}$ para trabalhadores masculinos e $30 \mathrm{ig} /$ $\mathrm{dL}$ para mulheres. A American Conference of Governmental Industrial Hygienists ${ }^{35}$ dos Estados Unidos da América do Norte adotou um LTB de $50 \mathrm{ig} / \mathrm{dL}$ de chumbo no sangue e no guideline de 2001 o indicador de dose interna atualizado é de $30 \mathrm{ig} / \mathrm{dL}^{25}$, fazendo a ressalva que mulheres em idade fértil com plumbemia superior a $10 \mathrm{ig} /$ $\mathrm{dL}$ podem gerar crianças com chumbo sangüíneo de $100 \mathrm{ig} / \mathrm{dL}$, as quais podem desenvolver sérias lesões no desenvolvimento cognitivo. No Brasil o Ministério do Traba$\mathrm{Iho}^{36}$ adota como valor de referência o $\mathrm{Pb}$ -
$S$ de até $40 \mathrm{ig} / \mathrm{dL}$ e para exposição ocupacional o IBMP (Índice Biológico Máximo Permitido) de $60 \mathrm{ig} / \mathrm{dL}$. Neste estudo constatamos que $43,5 \%$ dos trabalhadores do grupo de sinterização, 36,6\% da fusão, $32,6 \%$ da refinaria e $23 \%$ da manutenção possuíam $\mathrm{Pb}-\mathrm{S}$ superior a $40 \mathrm{ig} / \mathrm{dL}$, considerado como normal pela legislação brasileira e igual ou superior ao LTB estabelecido em diversos países, enquanto 12,2\% dos trabalhadores da área de fusão, 10,2\% da de refinaria e $7 \%$ da de sinterização, totalizando 12 trabalhadores nos três grupos, revelaram $\mathrm{Pb}-\mathrm{S}$ superior a $50 \mathrm{ig} / \mathrm{dL}$, valor de chumbo sangüíneo mais elevado do que o estabelecido como LTB pela Organização Mundial da Saúde ${ }^{25}$ e a ACGIH dos Estados Unidos da América do Norte.

No que concerne ao bioindicador de efeito, concentração urinária de ALA, observamos que $85,4 \%$ dos trabalhadores da área de Sinterização e $81,6 \%$ da Refinaria, possuíam ALA-U superior a $4,5 \mathrm{mg} / \mathrm{g}$ creat. estabelecido como valor de referência pela legislação brasileira ${ }^{36}$, enquanto que $36,6 \%$ dos indivíduos da de sinterização, $48,8 \%$ da de fusão e $30,6 \%$ da de refinaria apresentaram ALA-U superior a $10 \mathrm{mg} /$ $g$ creat., adotado pela legislação brasileira como Índice biológico Máximo Permitido (IBMP).

A Figura 2 apresenta a corrrelação entre a plumbemia e a concentração urinária de ALA nos grupos controle e nos diversos grupos de trabalhadores da metalúrgica. modelo matemático que melhor descreve esta correlação é equação quadrática [ALA = $\left.0,007^{*}(\mathrm{~Pb}-\mathrm{S})^{2}-0,111^{*}(\mathrm{~Pb}-\mathrm{S})+4,51\right] \mathrm{com}$ coeficiente de correlação $\left(r^{2}\right)$ de 0,249 . O teste t para significância do índice de correlação demonstrou que esta é uma correlação positiva e significativa $(p<0,05)$ entre os indicadores de exposição e de efeito. Esses dados estão de acordo com os resultados de outros estudos 2 , 17, 19, 22 , havendo uma inflexão nas concentrações de $\mathrm{Pb}$-S superiores a 40 ig/dL. Utilizando este modelo para prever a concentração de ALA-U a partir do $\mathrm{Pb}-\mathrm{S}$ de $40 \mathrm{ig} / \mathrm{dL}$, por exemplo, seria $11,3 \mathrm{mg} / \mathrm{g}$ creat.; concentração esta acima do IBMP para o chumbo inorgânico (10 mg/g creatinina).

Para a ZnPP os resultados mais elevados foram obtidos para os grupos da sinterização, fusão e refinaria; corroboran- 
do os achados a partir dos dados do ALA-U.

É possível concluir, portanto, que os trabalhadores com maior risco de exposição ocupacional ao chumbo na planta da metalúrgica estudada são representados pelos grupos da Fusão, Sinterização e Refinaria, alguns deles com elevadas concentrações de chumbo no sangue e importantes alterações na concentração de ZnPP sangüíneo e ALA urinário.

Apesar da constatação de considerável redução nos níveis de $\mathrm{Pb}-\mathrm{S}$ nos trabalhadores expostos em todo o mundo, motivando inclusive a revisão dos LTB's estabelecidos na legislação de saúde ocupacional de diversos países, mais recentemente tem sido enfatizada a importância da toxicidade subclínica do chumbo, demonstrando que concentrações de chumbo no sangue mesmo abaixo dos LTB's adotados atualmente, podem desencadear importante toxicidade como nefropatia, neurotoxicidade central e periférica, efeitos sobre a reprodução, câncer renal e hipertensão arterial. Por outro lado, a associação de outros metais, como arsênico, cádmio e zinco, presentes em de- terminados tipos de minério, como a galena, utilizada pela metalúrgica avaliada neste estudo, pode aumentar o risco de toxicidade do metal, como também influenciar na cinética de produção de determinados indicadores biológicos utilizados na monitorização dos trabalhadores expos-

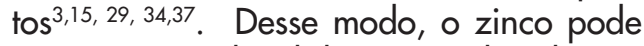
ativar a ácido delta-aminolevulínico desidratase (ALA-D), influenciando na produção de ALA-U22, o cádmio pode aumentar a nefrotoxicidade do chumbo, bem como outros fatores, como o consumo de álcool, que influencia na produção de alguns destes indicadores ${ }^{38}$.

Atualmente esta metalúrgica encontra-se desativada, mas deixou um passivo ambiental de mais de 50.000 toneladas de escória com contaminação superior a $3 \%$ de chumbo, ocasionando a contaminação ambiental e a consequüente contaminação da população da região $0^{6,30}$, merecendo estudos de monitorização ambiental e avaliação da população circunvizinha por longo período.

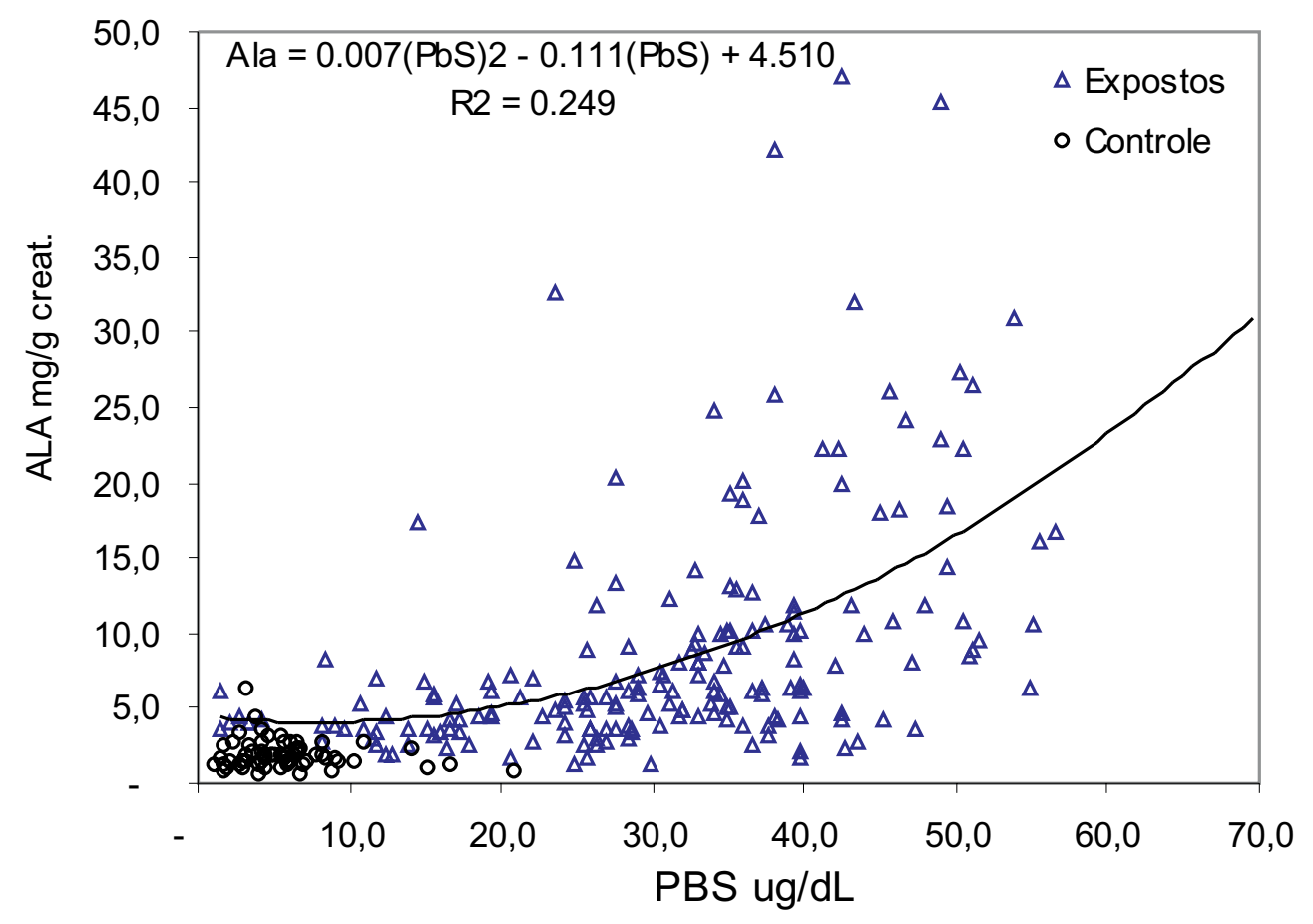

Figura 2 Correlação entre PB-S e ALA urinário dos grupos controle e dos trabalhadores expostos ao chumbo. 


\section{Referências Bibliográficas}

1.Wineger, D.A.; Levy, B.S.; Andrews, J.S.; Landrigan, P.J.; Scruton, W.H. \& Krause, M.J. Chronic Occupational Exposure to Lead: An Evaluation of the Health of Smelter Workers. J. Occup. Med., 1977, 19:603-607.

2. Lee, B.K. Occupational Lead Exposure of storage Battery Workers in Korea, Br. J. Ind. Med. 1982, 39: 283-289.

3. Landrigan, P.J. Current Issues in the Epidemiology and Toxicology of Occupational Exposure to Lead. Environmental Health Perspectives 89: 61-66, 1990.

4. Apostoli, P. \& Alessio, L. II Piombo Negli Anni 90: "Nuove" Regole per il Piú "Vecchio" Tossici Ambientali? Med. Lav. 1992; 82(6)539-556.

5. Landrigan, P.J.; Gehlbach, B.F.; Rosenblum, J.M. Shoults, R.M.; candelaria, W.F.; Barthel, J.A., Liddle, A.L.; Shrek, N.W.; Staehling, J.F. \& Sanders. Epidemic Lead Absorption Near an Ore Smelter; The Role of Particular Lead. N. Engl. J. Med. 1975, 283:123-129.

6. Carvalho, F.M.; Silvany-Neto, A.M.; Tavares, T.M.; Lima, M.E.C. \& Waldron, H.A. Intoxicação por Chumbo entre Crianças de Santo Amaro, Bahia. Bol. of. Sanit. Panam. 1986, 100(9).

7. González-Cassio, T; Ptereson, KE; Sanin, $\mathrm{LH}$; Fishbein, E; Palazuelos, E; Aro, A; Hernández-Avila, $M$ \& Hu, H. Decrease in Birth Wheight in Relation to Maternal Bone-Lead Burden. Pediatrics Vol. 100 No. 5 Novembro 1997.

8. Logerkvist, BJ; Ekersrydh, S; Englyst, V; Nordberg, GF; Söderberg, HA \& Wiklund, DE. Increased Blood Lead and Decreased Calcium Levels During Pregnancy : A Prospective Study of Swedish Women Living near a
Smelter. Am. J. Ped. Health . September 1996, Vol. 86 No. 9.

9. Menezes-Filho, J.A; Onofre, C.R; Bastos, C.A.C.; Lopes, A.A. \& Carvalho, W.A. The Association of the Social Economical Status and Blood Lead Level in Children of an Urban Area of Salvador - Bahia, Brazil. Book of Abstratcts of The $4^{\text {th }}$ International Symposium on Biological Monitoring in Occupational and Environmental Health. September 23-25, 1998 Seoul, Korea

10. Organizacion Panamericana de la Salud (OMS) Criterios de Salud Ambiental 3 - Plomo, Washington, OMS. 1979.

11. Correy, G. \& Galvão, L.A.C. Plomo, Serie Vigilancia 8, Metepec. Organizacion Panamericana de la Salud, 1985.

12. Gerhardsson, L.; Chettle, D.R.; englyst, V.; Nordberg, G.F.; Nyrlin,; Scott, M.C. \& Todd, A.C. Kidney Effects in Long Term exposed Lead Smelter Workers. Br. J. Industry. Med. 1992, 49: 186192.

13. Dalton, C.B.; McCammon, J.B.; Hoffman, R.E. \& Baron, R.C. Blood Lead Levels in Radiator Repair Workers in Colorado. J. Occup. Environ. Med. 1977, 35:58-62.

14. Goyer. R.A. Toxic Effects of Metals. In: Amdur, M.O.; Doull, J.; Klaassen, C.D. Casarett and Doull's Toxicology, the Basic Science of Poisons, $4^{\text {th }}$ Ed., New York, McGraw-Hill, 1991; 623-680.

15. Landrigan, P.J. Toxicity of Lead at Low Dose. Brit. J. Industr. Med. 1989, 46:593-596.

16. Cramér, K. \& Selander, S. Studies in Lead Poisoning. Brit. J. Industr. Med. 1965, 22:311-314. 
17. Selander, S. \& Cramer, K. Interrelationships Between Lead in Blood, lead in Urine and ALA in Urine during Lead Work. Brit. J. Industr. Med. 1970 27:28-39.

18. Waldron, H.A. Correlation Between Some Parameters of Lead Absorption and Lead Intoxication. Brit. J. Industr. Med. 1971, 28:195-199.

19. Zielhius, R.L. Interrelationships of Biochemical Responses to the Absorption of Inorganic Lead. Arch. Environ. Health. 1971, 23:299-310.

20. Tolla, S.; Hernberg, S.; Asp, S. \& Nikkaren, J. Parameters Indicative of Absorption and Biological Effect in New Lead Exposure: a Prospective Study. Brit. J. Industr. Med. 1973, 30:134-141.

21. Hammond, P.B.; O'Flaherty, E.J. \& Gartside, P.S. The Impact of Air-Lead on Blood Lead in Man - a Critique of the Recent Literature. Fd. Cosmet. Toxicol. 1981, 19:631-638.

22. Moore, M.R. Merredith, P.A. \& Goldberg, A. Lead and Heme Biosynthesis . In: Singhal, R.L. \& Thomas, J.A. Lead Toxicity, Urban \& Schwarzenberg, Baltimore, 1980; 78117.

23. Pruszkowska, E.; Carnick, G.R. \& Slavin W. Blood Lead Dertermination with the Platform Furnace Technive. At Soectros. 1983, 4:59-61.

24. Tomokuni, K. \& Ogata, M. Single Method for Determination of Urinary d-Aminolevulinic Acid as an Index of Lead Exposure. Clin. Chem. 1972, 19(12):1534-6.

25. World Health Organization - Biological Monitoring of Chemical Exposure in the Workplace, Volume 1, Geneve, WHO, 1996; 112-131.

26. Kim, R.; Landringan, C.; Massmann, P.; Sparrow, D. \& Hu, H. Age and Secular Trends in Bone Lead Levels in
Middle-Aged and Elderly Men: Threeyear Longitudinal Follow-up in the Normative Aging Study. 1997, Am. J. Epidemiol. 146:586-91

27. Torra, M.; Rodamilans, M.; Montero, F.; Farre, C. \& Carbella, J. Estudio de la Exposicion al Plomo en la Populacion de Barcelona: Evolucion Cronologica entre 1984 y 1995. Med. Clin. Barc. 1997, 108:601-3.

28. Mahaffey, K.R.; Amnest, J.L.; Roberts, J.H. \& Murphy, R.S. Estimates of Blood Lead Levels: United States 19761980. Association with Selected Demographic and Socioeconomic Factors. N. Engl. J. Med. 1982, 307:573-579.

29. Gerhardsson, L.; Kazantzis, G. \& Schutz, A. Evaluation of Selected Publications on Reference Values for Lead in Blood. Scand. J. Work Environ. Health. 1996, 22:325-31.

30. Fernícola, N.A.G.G. \& Azevedo, F.A. Níveis de Chumbo e Atividade da Desidratase do Ácido dAminolevulínico (d-ALA-D) no Sangue da População da Grande São Paulo, Brasil. Rev. Saúde Publ. 1981, 15: 272-82.

31. Suga, R.S.; Fischinger, A.J. \& Knorch, F.W. Establishment of Normal Values in Adults for Zinc Protoporphyrin (ZnPP) Using Haematoflurometer: Correlation with Normal Blood Values. Am. Ind. Hyg. Assoc. J. 42:637-642, 1991.

32. L'abbate, N. Cassa, F.; Rana, F.; Gagliardi, T. \& Giacomoantonio, A. Blood Lead and Erythrocyte Protoporphyrin Levels in the General Population of an Area in Southern Italy. Med. 1991, Lav. 82(4):336-340.

33. Baker, E.L.; Landrigan, P.J; Barbour, A.G.; Cox, D.H.; Folland, D.S.; Liogo, R.N. \& Throckmorton, J. Occupational Lead Poisoning in the United States: Clinical and Biochemical Findings Related to Blood Lead Levels. Brit. J. Ind. Med. 1979, 36: 314-322. 
34. Lundstron, N.G.; Nordberg, G.; Englyst, J.; Gerhardsson, L.; Hagmar, L.; Jin, T.; rylander, L. \& Wall, S. Cummulative Lead Exposure in Relation to Mortality and Lung Cancer Morbidity in a Cohort of Primary Smelter Workers. Scand. J. Work. Environ. Health. 1997, 23:2430.

35. American Conference of Governmental Industrial Hygienists (ACGIH). Threshold Limit Values for Chemical Substances and Physical Agents and Biological Exposure Indices, Cincinnati, ACGIH, 2001.

36. Brasil Ministério do Trabalho, Portaria $\mathrm{N}^{\circ}$ 24, de 29.12.94. Norma Regulamentadora $N^{\circ}$ 7. Diário Oficial da União de 30.12.94, Brasilia. .
37. Cocco, P.; Hua, F.; Boffeta, P.; Carta, P.; Flore, V.; Onnis, A.; Picchiri, G.F. \& Colin, D. Mortality of Italian Lead Smelter Workers. Scand. J. Work Environ. Health. 1997, 23: 15-23.

38. Candela, S,; Piccinini, R. Viappiani, F. \& Lari, U. Influenza Dell'Alcool sul Comportamento degli Indicatori di Dose e di Effeto in Lavoratori Esposti a Piombo Inorganico: Comportamento Inateso della ZnPP. Med. Lav. 1991, 82:523-541.

39. Anjos, JASA. Estratégias para remediação de um sítio contaminado por metais pesados - Estudo de Caso. São Paulo, 1998, 157p. Dissertação (Mestrado) Escola Politécnica. Universidade de São Paulo. 